\title{
Pharmacogenetics of metformin response: a step in the path toward personalized medicine
}

\author{
Marc L. Reitman ${ }^{1}$ and Eric E. Schadt ${ }^{2}$ \\ ${ }^{1}$ Metabolic Disorders Department, Merck Research Laboratories, Rahway, New Jersey, USA. \\ ${ }^{2}$ Rosetta Inpharmatics LLC, a wholly owned subsidiary of Merck \& Co. Inc., Seattle, Washington, USA.
}

\begin{abstract}
Type 2 diabetes mellitus affects $9.6 \%$ of the adults in the United States and more than 200 million people worldwide. Diabetes can be a devastating disease, but it can now be treated with nine classes of approved drugs (insulins, sulfonylureas, glinides, biguanides, $\alpha$-glucosidase inhibitors, thiazolidinediones, glucagon-like peptide 1 mimetics, amylin mimetics, and dipeptidyl peptidase 4 inhibitors), in addition to diet and exercise regimens. Choosing which drug to give a patient is based on efficacy and also availability, cost, safety, tolerability, and convenience. Personalized medicine promises a path for individually optimized treatment choices, but realizing this promise will require a more comprehensive characterization of disease and drug response. In this issue of the JCI, Shu et al. make significant progress by integrating diverse data supporting the hypothesis that genetic variation in organic cation transporter 1 (OCT1) affects the response to the widely used biguanide metformin (see the related article beginning on page 1422). We discuss metformin, OCT1, pharmacogenetics, and how the integrative genomics revolution is likely to change our understanding and treatment of diabetes.
\end{abstract}

\section{Why study drug response pharmacogenetics?}

Variability in drug response, with respect to efficacy, tolerability, and safety, is a major issue for most drugs. Diabetes, like other complex diseases, is the product of many genes interacting with environmental factors. These interactions affect many pathways and even whole signaling networks in ways that cause disease. The promise of personalized medicine is that this complexity can be teased apart to refine the definition of disease, identify disease subtypes, and ultimately define biomarkers capable of discriminating between the patients most likely to benefit from a specific treatment and those unlikely to respond or likely to experience adverse events. One approach to this problem is to undertake pharmacogenetic studies to identify DNA variations in specific genes that are associated

Nonstandard abbreviations used: ADME, absorption, distribution, metabolism, and excretion; AMPK, AMP-activated protein kinase; KCNJ11, potassium inwardly rectifying channel, subfamily $\mathrm{J}$, member 11; OCT, organic cation transporter; TCF7L2, transcription factor 7-like 2.

Conflict of interest: The authors are both employees and stockholders of Merck \& Co.

Citation for this article: J. Clin. Invest. 117:1226-1229 (2007). doi:10.1172/JCI32133. with response to treatment (Figure 1A). There have been many successes using this straightforward genetic approach, particularly with respect to genes that encode drug-metabolizing enzymes such as cytochrome P450s (1). Other successful examples include the identification of variations in the gene vitamin $K$ epoxide reductase complex, subunit 1 (VKORC1, the target of the anticoagulant warfarin), associated with effective warfarin dosing (2), and variation in Niemann-Pick disease, type C1, gene-like 1 (NPC1L1), associated with the response to treatment with ezetimibe, a cholesterol uptake inhibitor (3).

We use the term "pharmacogenetics" for pure gene-focused genetic approaches, typically involving testing candidate genes. "Pharmacogenomic" studies involve larger-scale genome-wide association approaches or integration of functional genomic (RNA expression) or other dense data sets to predict drug response. Establishing an association between a variation in DNA and drug response will not necessarily provide mechanistic understanding of the drug response phenotype. For example, if a DNA polymorphism associated with drug response is not near the gene in question or is in a gene of unknown function, experimen- tation is required to identify the gene's mechanistic role in the response. Furthermore, each locus typically has a modest effect on complex phenotypes such as drug response and must be subjected to prospective replication studies in independent cohorts to ensure that the polymorphism is truly a predictor of response. In this issue of the JCI, Shu et al. (4) take a multipronged approach, using cell-based experiments, in vivo studies in mice, and in vivo human trials to demonstrate the relevance of DNA variations in organic cation transporter 1 (OCT1, also known as solute carrier family 22, member 1 [SLC22A1]) to metformin response (Figure 1B). This approach avoids pinning hopes on any single approach, instead providing multiple independent lines of evidence supporting the hypothesis that OCT1 is required for metformin action and that genetic variation in OCT1 may have a significant impact on response to the drug.

\section{Metformin}

Metformin and phenformin were discovered in the 1920s in a search for guanidinecontaining compounds with antidiabetic activities. These biguanides were introduced clinically in Europe in the 1950s; phenformin was withdrawn in the 1970s due to its potential to induce lactic acidosis. Metformin was approved for use in the United States in 1995 (5). A major action of metformin is suppression of hepatic glucose production. In addition to its efficacy in lowering glucose levels, metformin has the clinical advantages of inducing mild weight reduction and only a minimal risk of hypoglycemia, but it has some gastrointestinal side effects and, very rarely, causes lactic acidosis. Metformin is often the first drug used to treat newly diagnosed type 2 diabetic patients. In 2006, it accounted for $37 \%$ of the non-insulin diabetes prescriptions in the United States (IMS National Prescription Audit database; http:// www.imshealth.com/ims/portal/front/ articleC/0,277,6599_18731_40044561,00. 
A The classic pharmacogenetic approach
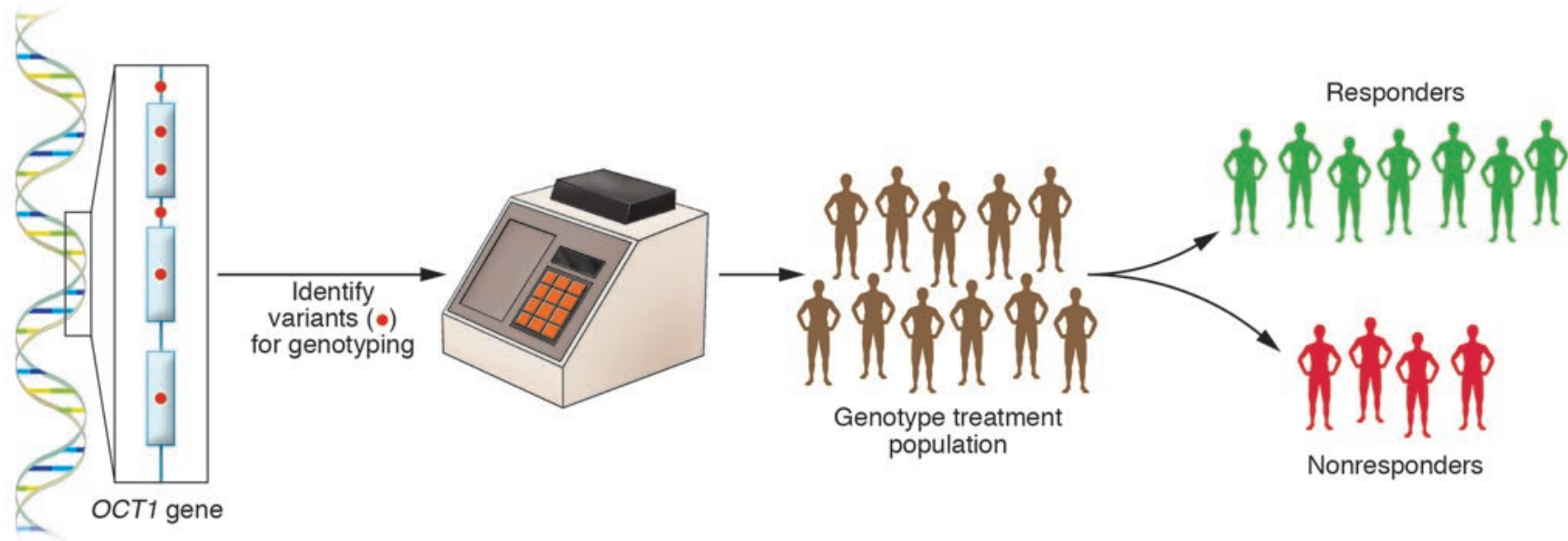

Nonresponders

Genome

B A more integrative pharmacogenomic approach

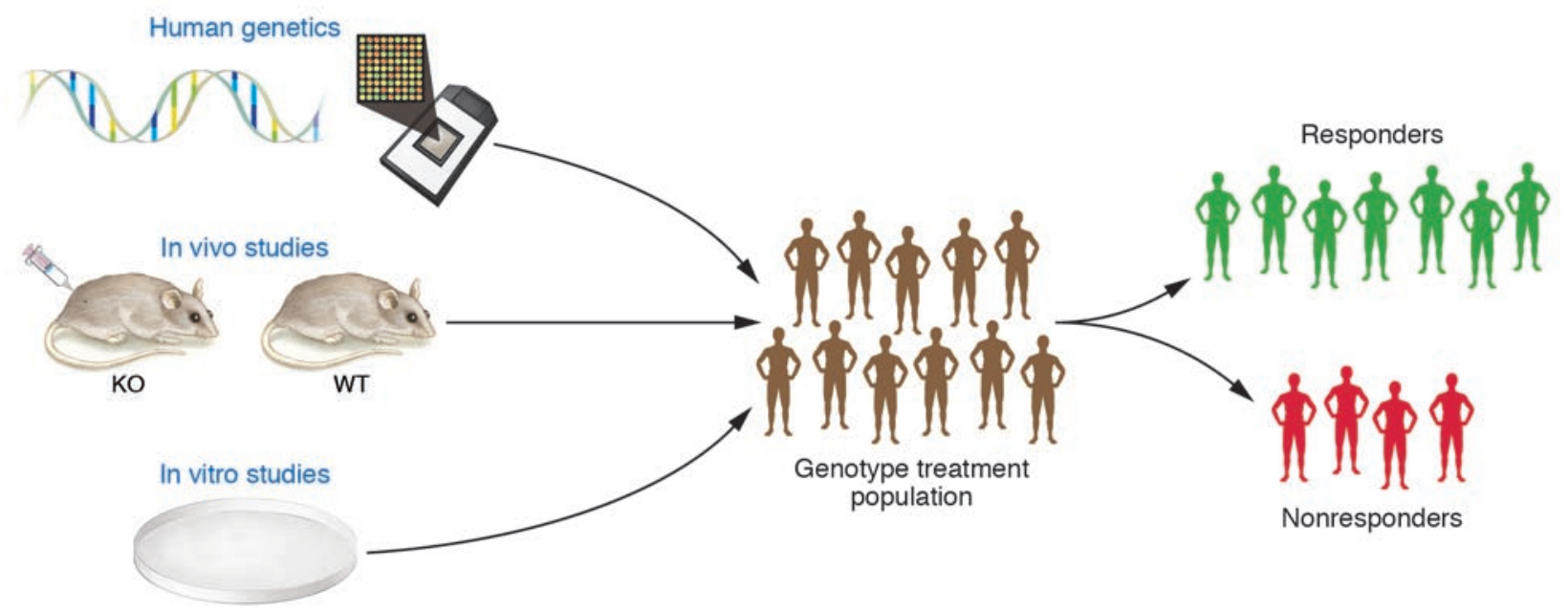

Figure 1

Elucidating the complexity of drug response. (A) A classical pharmacogenetic approach to identifying genes associated with drug response involves testing DNA variations in candidate genes for association to drug response. Variations in cytochrome P450s and drug targets such as VKORC1 (target of warfarin) have been identified that explain variation in drug response. Individual genotypes corresponding to such variations can be used to distinguish responders from nonresponders or rapid from slow metabolizers or those at risk of adverse events. (B) Pharmacogenomic studies involve larger-scale genome-wide association approaches or the integration of a diversity of genetic, functional genomic, and other data to elucidate the complexity of drug response. In this issue of the JCl, Shu et al. (4) employed cell-based experiments, in vivo studies in mice, and in vivo human trials to identify OCT1 as a gene associated with response to treatment with metformin. The use of multiple experimental approaches provided more convincing results than could a single genetic study in implicating OCT1 as a metformin response gene.

html). Metformin's effect is more durable than that of the sulfonylurea glyburide, which is also prescribed to help control blood sugar levels (6).

The molecular target of metformin, like that of almost all drugs of its vintage, was not known when it entered clinical use, and, while there has been much progress in defining its physiologic effects, the direct target remains unknown. In isolat- ed hepatocytes, metformin action requires the enzyme AMP-activated protein kinase (AMPK), a master sensor, integrator, and regulator of cell and body energy homeostasis (7). AMPKs are activated by an increased AMP/ATP ratio and by upstream kinases (e.g., LKB1) (8). AMPK activation affects many pathways, generally causing conservation and generation of ATP (8). How metformin activates AMPK, such as through an upstream kinase or by altering the cell's energy status and thus AMP/ATP ratio, is not yet clear.

\section{OCTs and metformin}

Wang et al. previously showed that metformin levels were greatly reduced in liver and intestine of $\mathrm{Oct}^{-/-}$mice, indicating that Oct 1 is the major mechanism for metformin entry into hepatocytes and entero- 
cytes (9). Kidney and circulating metformin levels were comparable in $\mathrm{Oct}^{-/-}$and control mice, consistent with more recent data suggesting that Oct 2 and not Oct 1 is the major renal metformin transporter and the determinant of its renal secretion (10). In addition, human genetic variants of OCT1 have been characterized, including a number that reduced transporter activity (11).

In their study reported in this issue of the JCI, Shu et al. (4) first use various cells to demonstrate a correlation between OCT1 activity and the ability of metformin to activate AMPK. Next, Oct1 $1^{-/-}$mice dosed with metformin were shown to have plasma exposures and kinetics similar to those of control mice, but with four-fold lower liver accumulation (at 1 hour) and decreased liver AMPK activation. Mice fed a high-fat diet for eight weeks were then treated with metformin for five days. Metformin reduced the fasting glucose level in control but not Oct $1^{-/-}$mice. Shu et al. then studied the function of known human OCT1 variants, identifying seven with reduced metformin uptake, which generally also resulted in reduced AMPK activation. Finally, humans heterozygous or homozygous for severely reduced function alleles of OCT1 were studied for their response to metformin. Oral glucose tolerance tests at baseline were comparable in the two groups, while after two doses of metformin, individuals with reducedfunction alleles showed slightly higher glucose levels.

Very recently, Shikata et al. also investigated the role of OCT1 in the clinical response to metformin (12). Patients were assigned responder $(n=24)$ or nonresponder $(n=9)$ status based on their response to more than three months of treatment with metformin. They observed no remarkable differences in the prevalence of OCT1 polymorphisms between the two groups. There are a number of possible reasons why these two studies $(4,12)$ seemingly reach different conclusions. Of major importance is the subject identification approaches employed: Shu et al. (4) used OCT1 genotype, while Shikata et al. (12) used metformin response (to which the relative contribution of OCT1 is unknown). The studies were done in different ethnic groups having different background genetics. While the number of subjects in each study was small, the numbers in the Shikata study were minimal given the hypothesis of allele frequency shifts between the responder and nonresponder groups. Neither group measured plasma metformin levels. Shu et al. measured the acute effect of the drug, while Shikata et al. used chronic efficacy of the drug as a measure. This last difference may also be crucial, since most of the variants tested by Shu et al. were not nulls, so one might expect a delay in liver uptake, measurable at specific times after dosing, but one that was possibly not of chronic importance. While Shu et al.'s data are tantalizing, it must be stressed that, as with all human genetic studies, a prospective, larger-scale replication of this study is required before the OCT1 genotype can be considered a validated predictor of clinical response to metformin.

There are also other questions about the pharmacogenetics of metformin and OCT1 and OCT2. Is the incidence of lactic acidosis (estimated at 1 in 33,000 treated patients; ref. 5) affected by these genes? The OCT1 $\mathrm{S} 14 \mathrm{~F}$ allele is a hyperfunctioning allele (11). Does it cause faster equilibration between the liver and plasma metformin compartments, increase the measured response to metformin, or have no measurable effect? Does the OCT2 genotype affect plasma levels of metformin?

\section{Genetics and pharmacogenomics}

We are at the dawn of the age of pharmacogenomics and personalized medicine and ever closer to achieving the "\$1,000 genome." What does this mean for diabetes?

Forward genetic approaches (i.e., starting from phenotype and identifying the genetic cause) to dissecting mendelian forms of diabetes have been hugely successful in identifying a small subset of diabetic patients in whom rare, highly penetrant mutations of a single gene cause their diabetes (13). While common variants of these genes that make a small contribution to polygenic diabetes may also exist (13), the variants causing monogenic diabetes have limited utility in pharmacogenetics due to their low allele frequency. The vast majority of type 2 diabetes patients have polygenetic forms of the disease that typically also require a permissive environment (e.g., obesity, sedentary lifestyle, advancing age, etc.) to be penetrant. Each locus contributes a small amount of risk (odds ratios typically ranging from 1.1- to 1.5-fold), so large cohorts are needed to identify the at-risk alleles. Some of the loci identified to date include transcription factor 7-like 2 (TCF7L2) (14), calpain 10 (CAPN10) (15), peroxisome proliferator-activated receptor $\gamma(P P A R G)(16)$, and potassium inwardly rectifying channel, subfamily J, member 11 (KCNJ11) (17). However, the pace of gene identification is increasing due to the availability of large-scale databases of genetic variation and advances in genotyping technology. A recent genome-wide study identified solute carrier family 30, member 8 (SLC30A8), a $\beta$ cell $\mathrm{Zn}$ transporter, and two other genomic regions as additional diabetes risk loci (18).

Pharmacogenetics differs from more classical genetic approaches in that there must be an interaction between gene and drug therapy, as opposed to a more straightforward association to disease. Drug-genome interactions can occur in a number of ways. Genetic variation in the direct molecular target of a drug class is one obvious type of pharmacogenetic interaction. For example, this appears to be the case for KCNJ11 (a $\beta$ cell K channel), with one allele apparently predicting reduced success of sulfonylurea treatment (19). Another class of genes are those involved in drug ADME - a bsorption, distribution (such as OCT1), metabolism, and excretion. In general, ADME genes can affect the action of many drugs, independent of their intended molecular target. Another group of genes affecting drug action are those affecting the underlying disease. However, in one case where this interaction was studied in order to see whether TCF7L2 predicted the response to metformin, it was not found (20). Like diabetes itself, drug response is a complex trait, involving many genes interacting with environmental conditions. Therefore, more integrative approaches, rather than genetic association approaches alone, are needed to effectively dissect drug response traits (Figure 1). In their current study, Shu et al. (4) provide an excellent example of the type of integrative approach that will not only identify functional variants in genes that associate with response but also elucidate metabolism and signaling pathways and mechanisms of action, increasing confidence in the results.

What are the cautions and pitfalls of pharmacogenetics that we must keep in mind? The level of complexity can be staggering, potentially requiring huge cohorts and commensurate resources to perform investigations. What if the drug effect interacts non-independently with more than one locus and also non-independently 
with other traits (for example, metformin works better in younger patients; ref. 21). The required size of a study to properly delineate these effects quickly becomes prohibitive, so currently the best possible approaches involve simplifying assumptions of non-interdependence or underpowered analyses.

What are the benefits of personalized medicine? Integrative pharmacogenomics studies, such as that of Shu et al. (4), are likely to reveal the true diversity of responders and therefore lead to personalized treatment regimens that ensure administration of the right drug for the right person at the right time. Knowing the biology of the polymorphisms in the genes defining the metabolic and signaling pathways associated with a drug and the disease it treats is potentially of immediate benefit to the patient. Metformin is typical of diabetes drugs, with patients showing a range of responses. Tailoring therapeutic choices to the individual should allow lower and better-tolerated doses of drugs that are simultaneously safer and more efficacious. One could eliminate trial-and-error periods of switching among the nine classes of diabetes drugs. Having determined an appropriate drug class, choice of the most suitable drug from that class could be guided by pharmacogenomic data. Most exciting, having the ability to match individuals to safe, well-tolerated, efficacious drugs could lead to paradigm shifts. For example, rather than waiting for frank diabetes to develop before starting treatment, pharmacogenomics could foster the acceptance of treating people with impaired glucose tolerance or impaired fasting glucose levels (21), preventing development of diabetes and its sequelae. Shu et al.'s work brings us a step closer to realizing this promise.

Address correspondence to: Marc L. Reitman, Merck Research Laboratories, PO Box 2000, RY80M-213, 126 East Lincoln Avenue, Rahway, New Jersey 07065-0900, USA. Phone: (732) 594-4609; Fax: (732) 594-3337; E-mail: marc_reitman@merck.com.

1. Eichelbaum, M., Ingelman-Sundberg, M., and Evans, W.E. 2006. Pharmacogenomics and individualized drug therapy. Annu. Rev. Med. 57:119-137.

2. Rost, S., et al. 2004. Mutations in VKORC1 cause warfarin resistance and multiple coagulation factor deficiency type 2. Nature. 427:537-541.

3. Simon, J.S., et al. 2005. Sequence variation in NPC1L1 and association with improved LDL-cholesterol lowering in response to ezetimibe treatment. Genomics. 86:648-656.

4. Shu, Y., et al. 2007. Effect of genetic variation in the organic cation transporter 1 (OCT1) on metformin action. J. Clin. Invest. 117:1422-1431. doi:10.1172/ JCI30558.

5. Bailey, C.J., and Turner, R.C. 1996. Metformin. N. Engl. J. Med. 334:574-579.

6. Kahn, S.E., et al. 2006. Glycemic durability of rosiglitazone, metformin, or glyburide monotherapy. N. Engl.J. Med. 355:2427-2443.

7. Zhou, G., et al. 2001. Role of AMP-activated protein kinase in mechanism of metformin action. J. Clin. Invest. 108:1167-1174. doi:10.1172/ JCI200113505.

8. Hardie, D.G. 2007. AMP-activated protein kinase as a drug target. Annu. Rev. Pharmacol. Toxicol. 47:185-210.

9. Wang, D.S., et al. 2002. Involvement of organic cation transporter 1 in hepatic and intestinal distribution of metformin. J. Pharmacol. Exp Ther. 302:510-515.

10. Kimura, N., et al. 2005. Metformin is a superior substrate for renal organic cation transporter OCT2 rather than hepatic OCT1. Drug Metab. Pharmacokinet. 20:379-386.

11. Shu, Y., et al. 2003. Evolutionary conservation predicts function of variants of the human organic cation transporter, OCT1. Proc. Natl. Acad. Sci. U. S. A. 100:5902-5907.

12. Shikata, E., et al. 2007. Human organic cation transporter (OCT1 and OCT2) gene polymorphisms and therapeutic effects of metformin. J. Hum. Genet. 52:117-122.

13. O'Rahilly, S., Barroso, I., and Wareham, N.J. 2005. Genetic factors in type 2 diabetes: the end of the beginning? Science. 307:370-373.

14. Grant, S.F., et al. 2006. Variant of transcription factor 7-like 2 (TCF7L2) gene confers risk of type 2 diabetes. Nat. Genet. 38:320-323.

15. Tsuchiya, T., et al. 2006. Association of the calpain10 gene with type 2 diabetes in Europeans: results of pooled and meta-analyses. Mol. Genet. Metab. 89:174-184.

16. Altshuler, D., et al. 2000. The common PPARgamma Pro12Ala polymorphism is associated with decreased risk of type 2 diabetes. Nat. Genet. 26:76-80.

17. Gloyn, A.L., et al. 2003. Large-scale association studies of variants in genes encoding the pancreatic beta-cell KATP channel subunits Kir6.2 (KCNJ11) and SUR1 (ABCC8) confirm that the KCNJ11 E23K variant is associated with type 2 diabetes. Diabetes. 52:568-572.

18. Sladek, R., et al. 2007. A genome-wide association study identifies novel risk loci for type 2 diabetes. Nature. 445:881-885.

19. Sesti, G., et al. 2006. The E23K variant of KCNJ11 encoding the pancreatic beta-cell adenosine 5 '-triphosphate-sensitive potassium channel subunit Kir6.2 is associated with an increased risk of secondary failure to sulfonylurea in patients with type 2 diabetes. J. Clin. Endocrinol. Metab. 91:2334-2339.

20. Florez, J.C., et al. 2006. TCF7L2 polymorphisms and progression to diabetes in the Diabetes Prevention Program. N. Engl. J. Med. 355:241-250.

21. Knowler, W.C., et al. 2002. Reduction in the incidence of type 2 diabetes with lifestyle intervention or metformin. N. Engl. J. Med. 346:393-403. 